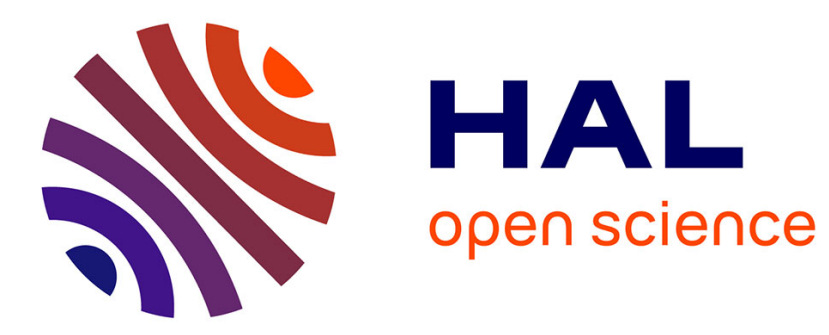

\title{
Improving the Accuracy of Industrial Robots by offline Compensation of Joints Errors
}

\author{
Adel Olabi, Mohamed Damak, Richard Béarée, Olivier Gibaru, Stéphane
}

Leleu

\section{- To cite this version:}

Adel Olabi, Mohamed Damak, Richard Béarée, Olivier Gibaru, Stéphane Leleu. Improving the Accuracy of Industrial Robots by offline Compensation of Joints Errors. IEEE International Conference on Industrial Technology, Mar 2012, Island of Kos, Greece. hal-00777382

HAL Id: hal-00777382

https://inria.hal.science/hal-00777382

Submitted on 17 Jan 2013

HAL is a multi-disciplinary open access archive for the deposit and dissemination of scientific research documents, whether they are published or not. The documents may come from teaching and research institutions in France or abroad, or from public or private research centers.
L'archive ouverte pluridisciplinaire HAL, est destinée au dépôt et à la diffusion de documents scientifiques de niveau recherche, publiés ou non, émanant des établissements d'enseignement et de recherche français ou étrangers, des laboratoires publics ou privés. 


\title{
Improving the Accuracy of Industrial Robots by offline Compensation of Joints Errors
}

\author{
Adel Olabi *, Mohamed Damak ${ }^{\dagger}$, Richard Bearee * Olivier Gibaru *and Stephane Leleu * \\ * Arts et Metiers ParisTech,CNRS,LSIS \\ 8 boulevard XIV, 59000 Lille, France \\ Email: adel.olabi@ensam.eu \\ $\dagger$ GEOMNIA, Lille, France \\ Email: m.damak@geomnia.eu
}

\begin{abstract}
The use of industrial robots in many fields of industry like prototyping, pre-machining and end milling is limited because of their poor accuracy. Robot joints are mainly responsible for this poor accuracy. The flexibility of robots joints and the kinematic errors in the transmission systems produce a significant error of position in the level of the end-effector. This paper presents these two types of joint errors. Identification methods are presented with experimental validation on a 6 axes industrial robot, STAUBLI RX $170 \mathrm{BH}$. An offline correction method used to improve the accuracy of this robot is validated experimentally.
\end{abstract}

\section{INTRODUCTION}

Industrial robots are usually used to realize industrial tasks like material handling, welding, cutting and spray painting. The mobility, flexibility and important work space of these robots allow using them in new fields of industry such as prototyping, cleaning and pre-machining of casts parts as well as end-machining of middle tolerance parts. These new applications require high level pose accuracy and to achieve a good path tracking. Unfortunately industrial robots are designed to have a good repeatability but not a good accuracy. Their repeatability ranges from 0.03 to $0.1 \mathrm{~mm}$ for small and medium sized robots and can exceed $0.2 \mathrm{~mm}$ for big ones. Meanwhile the accuracy is often measured to be within several millimeters [1]. This poor accuracy is caused by geometric factors, such as geometric parameters, joints offset errors and TCP definition, as well as by non-geometric factors such as compliancess, thermal effects gear, encoder resolution, gearboxes backlashes and kinematic errors.

Many fields of investigation are proposed to increase the accuracy of industrial robots like: robot calibration, process development and control system (see figure 1). Robot calibration improves the accuracy of positioning by reducing the deviation between the commanded pose and the real one.

The complete procedure of robot calibration basically consists of four stages: modeling, measurement, identification, and compensation [2]. In standard kinematic calibration, geometric errors are modeled and compensated; robot joints are assumed to be perfectly rigid [3]. In non-kinematic calibration, robot joints compliances and other non geometric errors are concidered [4]. In [5], authors have worked on modeling the Cartesian compliance of an industrial robot according to its joints compliances in order to analyze the system's stiffness.

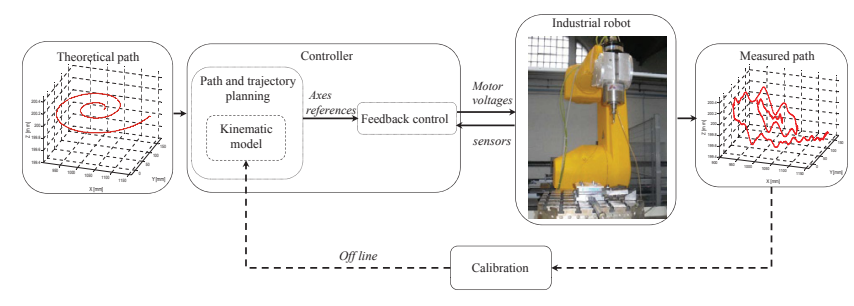

Fig. 1. functional chain of industrial robots

Regarding the control field, a large number of works have been done on trajectory planning, feedback control, system compensation and feedforward control [6] [7].

The work in this paper is situated in the field of nonkinematic calibration, as shown in figure 1 . We focus on two non-geometric errors: joints compliances and gearboxe kinematic errors. Different techniques could be used to compensate these errors. Typically, they can be classified in two categories:

- Model based method: in this method, each source of error must be identified and modeled by using a series of measurements of the real position of the end-effector. These models are integrated in a complex model of the robot to anticipate the positionning error in order to adjust the reference to correct this error [8] [9].

- Sensor based method: in this method the position error is measured by means of external position sensors. These sensors can be placed either in joint space or in Cartesian space. The measured error is injected then in the control loop to adjust the reference position in order to get the desired position of the end-effector [10].

The sensor based method offers a good position accuracy. However its implementation on an existing robot is difficult. Many problems could be encountered like the positioning of sensors on the robot structure and their integration in the control loop besides the extra cost due to the integration of these sensors. The model based technique allows to realize correction of the robot position without any change in the robot structure or in the control loop. In this paper, the model based technique is used to compensate two joints major errors: compliance errors and gearboxes kinematic error. For each kind of error two phases are necessary: identification 


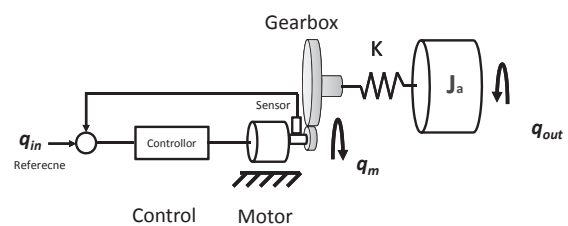

Fig. 2. model of robot joint wiht compliance

and modeling. The model of each error is integrated then in a trajectory planner, adapted for continous trajectories applications such as machining [11] [12], in order to realize an offline compensation of joints errors.

\section{JOINTS COMPLAINCES}

There are many sources of compliance in serial robots: base, joints and links, as well as an active compliance induced by the position control loop. Generally, the compliance of robot's links is negligible in comparison to joints compliance. Joints compliance consists of both axial and radial compliances. In this work, we take into account only the axial compliance, which is the dominating one. It is represented by an equivalent joint stiffness [13]. Due to joints compliance, external forces applied on the end-effector induce elastic deformations in robot's structure. The figure 2 presents a a model of a robot joint with the positioning of the sensor before the kinematic chain. These elastic deformations are not corrected by the controller because of the positioning of sensors on the motor axis and not on the joint axis. Therefore, to improve the accuracy of robots these deformations must be compensated.

\section{A. Identification of Joints stiffnesses}

The identification methods can be classified in two categories: global and local.

1) Global method: This method is based on measurements realized in the end-effector level. An example of setting up of this method is shown in figure 3 , where the external force $\mathbf{F}$ is measured by a force sensor. The end-effector position and the orientation deviation, due to $\mathrm{F}$, is measured by an external 3D measurement system, like a laser tracker. Thus, the relationship between the force $\mathrm{F}$ and the displacement vector $\delta \mathbf{X}$ is given by:

$$
\mathbf{F}=K_{c} \delta \mathbf{X}=J(\mathbf{q})^{-T} K_{J} J(\mathbf{q})^{-1} \delta \mathbf{X}, .
$$

where $J(\mathbf{q})$ is the Jacobian matrix and $K_{J}=$ diag $\left[K_{j 1}, K_{j 2}, \ldots, K_{j 6}\right]$ is the joint stiffness matrix. A least square solution could be done to calculate the stiffness matrix $K_{J}$.

The advantage of this method is the easiness of the installation. On the other side, it introduces many drawbacks:

- The results depend on the configuration used during the identification procedure,

- The position measurement, realized in the end-effector level, integerats the deformations of the robot links, which are not perfectly stiff.

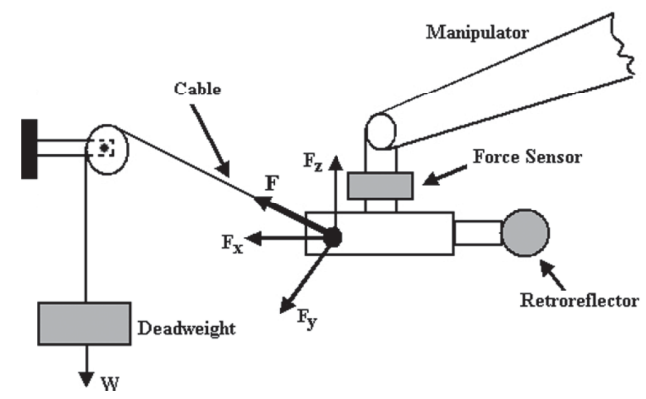

Fig. 3. Set up for the golabal identification method [13]

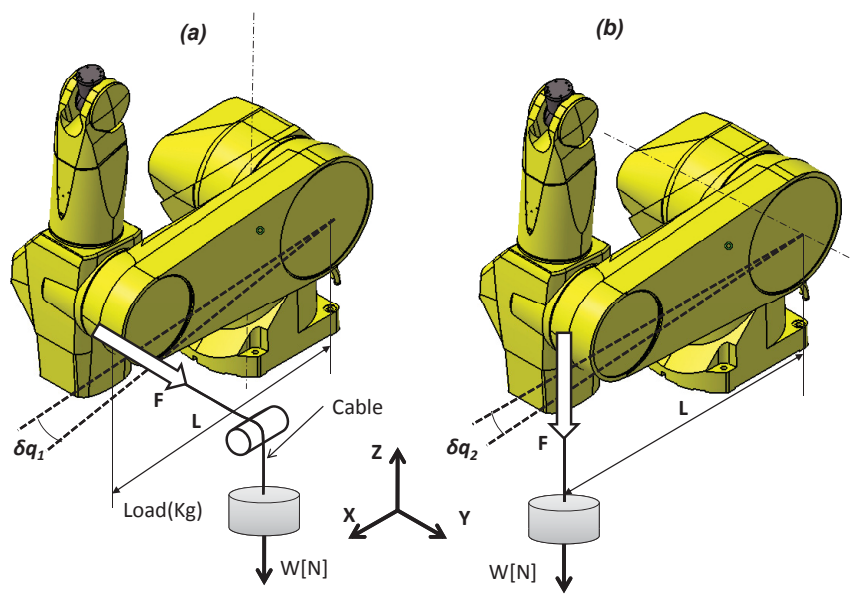

Fig. 4. Stiffness identification of joints 1 and 2

- The identification quality depends on the number of parameters to be identified at the same time.

To overcome these drawbacks we apply a local identification method, called axis by axis, where the stiffness of one joint is realized at each step.

2) local method: The only parameter to be identified in this approach is one joint stiffness. This stiffness is calculated by measuring the angular displacement resulted of an external torque applied on the joint. Our goal is to identify the stiffness of the six joints of our robot without disassembling it. This needs a special identification procedure to avoid the problem of stiffness coupling. This procedure changes according to the joint whose the stiffness is being identified. Where we chang the configuration of the robot for each joint stiffness identification, we measure the angular displacement by usinga Laser Tracker or an electronic level. We used a Laser Tracker for joints 1, 2 and an electronic level for joints 3,4,5 and 6 .

a) Joints 1,2: As shown in figure 4, the same configuration was selected for the stiffness identification of joints 1 and 2. An external force $\mathrm{F}$ is applied on the robot link to produce a torque on the joint axis. An angular displacement $\delta q$, is resulted of this torque; it is measured by the Laser Tracker. This operation is done for different external forces, then the stiffness is calculated by applying a linear interpolation model as follows: $F=K \delta q$. 


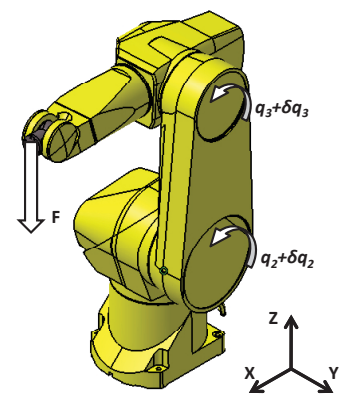

Fig. 5. Stiffness identification of joints 3

TABLE I

STIFFNESS OF ROBOT JOINTS

\begin{tabular}{|c|c|c|c|c|c|c|}
\hline Joint $N^{\circ}$ & 1 & 2 & 3 & 4 & 5 & 6 \\
\hline K[N.m/rad].10 & 0.204 & 0.85 & 0.57 & 0.49 & 0.12 & 0.005 \\
\hline
\end{tabular}

b) Joints 3,4,5 and 6: The same strategy, force/displacement, is applied for joints $3,4,5$ and 6 . However, in the cas of joint 3 identification, the application of an external force on the robot causes an angular displacement in joints 2 and 3 at the same time, as shown in figure 5 . For this reason two electronic levels are necessary: one is used as a reference to measure the angular displacement of axis 2 according to the vertical direction and another one to measure the combined angular displacements of joints 2 and 3. The positions of joints 2 and 3 are measured before applying the external torque, the relative angular position between these two joints before deformation is given by $\delta q=q_{3}-q_{2}$. After applying the force $\mathrm{F}$, the new values of the levels are $\hat{q}_{2}=q_{2}+\delta q_{2}$ and $\hat{q}_{3}=q_{3}+\delta q_{2}+\delta q_{3}$, where $\delta q_{2}$ and $\delta q_{3}$ are the angular displacement of the joints. The difference after deformation is $\hat{\delta} q=\hat{q}_{3}-\hat{q}_{2}$. Thereby, the angular displacement of joint 3 is given by:

$$
\begin{gathered}
\hat{\delta} q-\delta q=\left(\hat{q}_{3}-\hat{q}_{2}\right)-\left(q_{3}-q_{2}\right), \\
=\left(q_{3}+\delta q_{2}+\delta q_{3}+\delta q_{2}\right)-\left(q_{2}+\delta q_{2}\right), \\
=\delta q_{3}
\end{gathered}
$$

The strategy is used then to measure the stiffness of joints 4,5 and 6 . Figure 6 shows the positioning of the electronic levels during the identification procedure. The table I presents the values of the joints stiffnesses of our robot, founded by following the previous procedure.

\section{JOINTS KINEMATIC ERROR}

The movement transmission in a robot joints is not ideal. High ratio gearbox induces a kinematic error, noted $q_{\text {err }}$, which is given by the difference between the joint anglular position $q_{\text {out }}$ and the motor one $q_{i n}$, scaled by the ideal gear ratio [14] as follows:

$$
q_{\text {err }}=\frac{q_{\text {in }}}{\text { gear ratio }}-q_{\text {out }} .
$$

This kinematic error could limit the use of industrial robots for high accuracy demanding applications. This error can reach

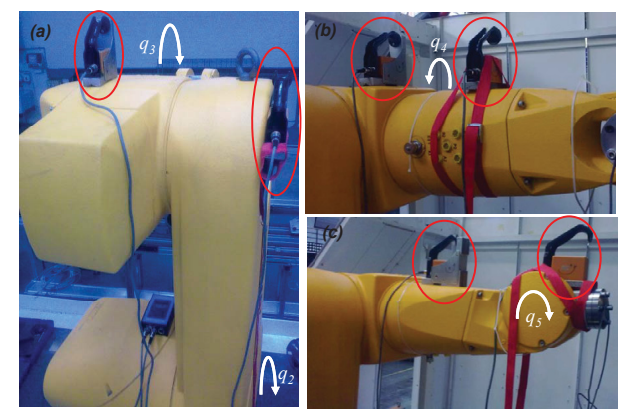

Fig. 6. Levels positionning for the identification of joints 3,4 and 5

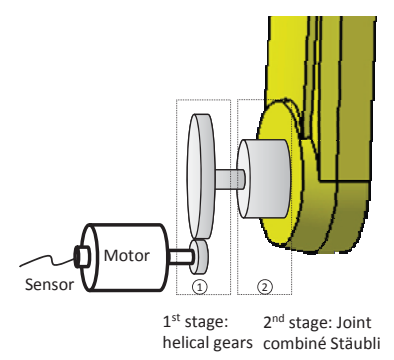

Fig. 7. Joint robot transmission system

$0.01^{\circ}$ for each joint and generate many $1 / 10$ of $\mathrm{mm}$ position error of the end-effector. The kinematic errors are due to defaults in the gears shape and inaccuracy in the joints and gearsboxes components assembly.

The three first joints of our robot are equipped with two stages of reduction system as shown in figure 7.

- The first level consists of two helical gears.

- The second level is a special reducer made by Staubli, similar to harmonic drives.

Thus, the kinematic error of each joint is composed of the defaults of the components of these two stages. Again, the objective is to measure the kinematic errors without disassembling the robot. Thereby, the identification is done by using measurements of the positions of robot links.

c) Identification protocol : To measure the kinematic error of a robot joint, we perform an angular displacement of this joint. Then the real position is measured by an external measurement system and compared to the rotary encoder commanded value. To avoid any dynamic effects, this operation is done by taking measurement in static positions. Thus, the identification of the kinematic error is done as follows:

- Measuring of the joint angular positions by an external system, for example a Laser Tracker, in a specified interval.

- Comparing these measures $q_{r}$ with the joint reference $q_{\text {theo }}$ to calculate the position error $\Delta q=q_{r}-q_{\text {theo }}$. This error cumulates the stiffness error $q_{s t i f f}$ and the kinematic error $q_{g e a r}$.

- Calculating the stiffness error by using robot parameters and then extract the kinematic error. 


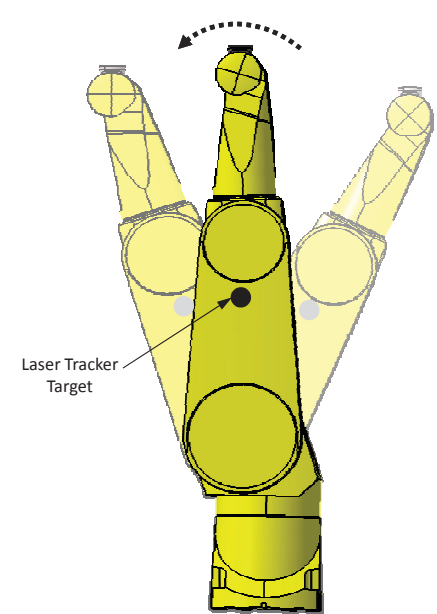

Fig. 8. Robot configuration for the measurement of joint 2 error

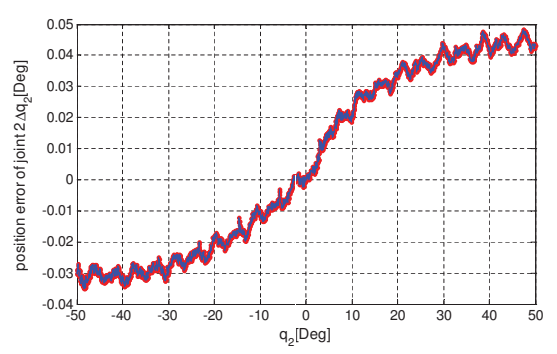

Fig. 9. Position error of joint 2

The Laser Tracker target is fixed on the robot link. This link is actuated with a specified angular step. The link motion is stopped after each step in order to measure automatically the position after a short stabilization time. It has to be noted that the laser tracker measures cartesian positions, so these positions must be converted to angular positions.

d) Joint 2: In this work the joint 2 of the robot is considered to validate this identification method. The selected configuration is shown in figure 8 . The robot joint is rotated from $q_{2}=-50^{\circ}$ to $+50^{\circ}$ (where $q_{2}=0$ is the vertical position) with a step of $0.05^{\circ}$. The waiting time after each step is one second before measuring the real position.

These measures are then converted to angular positions and compared to joint references for each step. The calculated position error is shown in figure 9. As mentioned before, this error cumulates:

- The stiffness error $d q_{s t i f f}$, about many $1 / 100$ of degree, is resulting from the joint deformation due to the residual torque applied on this joint. This residual torque is resulting from the difference between gravity and the compensation torques. In serial-type manipulators gravity effects are eliminated by systems of compensations [15]. For our robot, the gravity compensation is done in the level of joint 2 by a technique of spring suspension. . The residual torque is calculated by using our robot data in the test interval and it is shown in figue 10. This stiffness

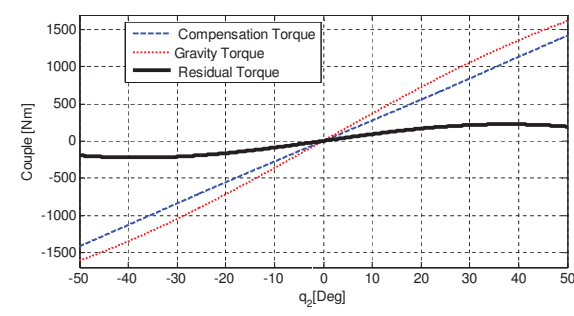

Fig. 10. Residual torque applied on joint 2

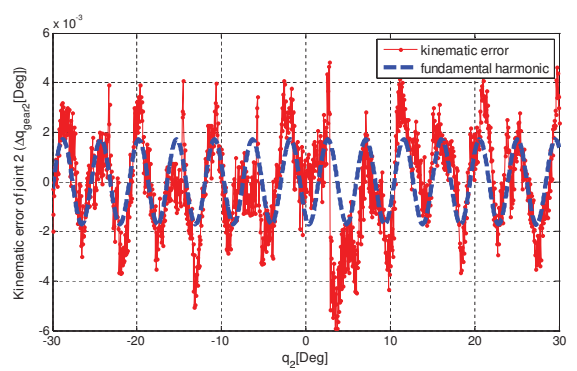

Fig. 11. Kinematic error of joint 2 and the fundamental harmonic

error is calculated then after having estimated the joint stiffness.

- The kinematic error $d q_{g e a r}$, about many 1/1000 of degree.

The kinematic error $\delta q_{\text {gear }}$ for joint 2 is calculated by subtracting the position error $\delta q$ from the stiffness error $\delta q_{\text {stiff }}$. This kinematic error is show in figure 11 .

The basic component of kinematic error for harmonic drive is due to a misalignment during assembly operation. The frequency of this component could be calculated according to drive input rotation. A typical signature of a drive error consists mainly of a fundamental harmonic occurring at a frequency of two cycles by input rotation [16]. The fundamental harmonic of the measured kinematic error according to the input rotation of the second level joint 2 transmission system confirms this explanation as shown in figure 11 .

\section{OFFLINE TRAJECTORY CORRECTION}

In this work we apply an offline strategy to eliminate the joints errors presented previously. This strategy is based on anticipating joints errors and correcting them by adjusting the position references of each joints as follows:

$$
Q_{\text {ref }}=Q_{\text {theo }}-\left(\Delta Q_{\text {stiff }}+\Delta Q_{\text {gear }}\right)
$$

where $\Delta Q_{\text {stiff }}$ is a vector of angular displacements due to joints deformations. This vector is calculated by using an elasto-static model based on geometric parameters, joints stiffness, components weights and gravity centre of these components. $\Delta Q_{\text {gear }}$ is a vector of joints kinematic errors. This correction strategy is applied in the trajectory planning stage, developed in previous works [11], where the joints references are modified according to the calculated errors. 


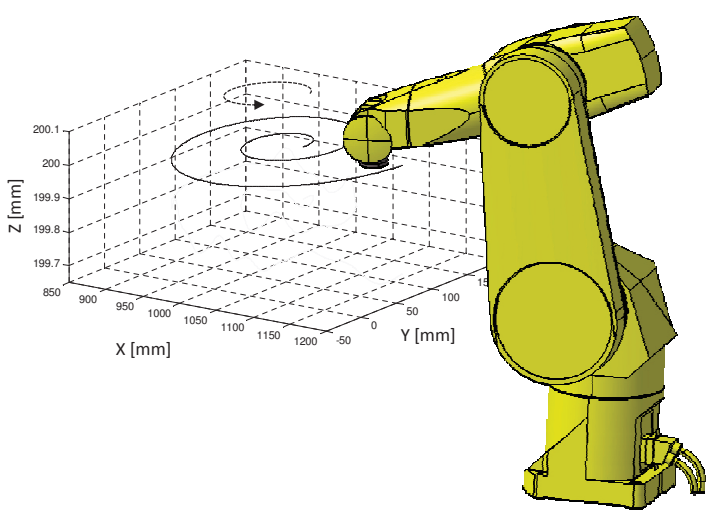

Fig. 12. Robot configuration during correction tests

TABLE II

ESTIMATION PARAMETERS OF Z ERROR

\begin{tabular}{|c||c||c|}
\hline Static Trajectories & $R_{a}[\mathrm{~mm}]$ & $R_{z}[\mathrm{~mm}]$ \\
\hline Without correction & 0.2032 & 0.2882 \\
\hline Stiffness correction & 0.0459 & 0.2097 \\
\hline Stiffness and kinematic correction & 0.0312 & 0.1502 \\
\hline
\end{tabular}

\section{A. Experimental tests}

For the sake of simplicity without losing generality we only apply the offline correction strategy on joint 2 . The configuration selected for these tests is presented in figure 12 . The tests are realized without any charge on the end-effector. In these conditions joint 2 errors are dominating.

The desired path is represented by a logarithmic spiral, given by $R=a \cdot e^{b \theta}$ where $a=24(\mathrm{~mm}), b=0.15(\mathrm{~mm})$ and $\theta=4 * \pi(\mathrm{rad})$. The spiral is placed in XY plan with $Z_{\text {robot }}=200 \mathrm{~mm}$. A Laser Tracker is used to measure the trajectory of the end-effector. To highlight the contribution of the correction of each error, we compensated firstly, the stiffness error then the kinematic error compensation is added.

e) Correction tests for quasi-static trajectories: To avoid any dynamic effects due to the inertia of the robot components, a low speed was selected, $\mathrm{S}=0.03 \mathrm{~ms}^{-1}$ and an acceleration of $\gamma=0.1 \mathrm{~ms}^{-2}$. The same trajectory was measured three times: without taking into account joint 2 errors then, with correction of joint stiffness error and finally by considering both, stiffness and kinematic errors.

The figure 13 shows the three trajectories measured during these tests. To quantify the correction of position of the endeffector in $\mathrm{Z}$ direction we use, by analogy with the roughness parameters in machining, the following parameters:

- The average value $R_{a}=\frac{1}{n} \sum_{k=1}^{n}\left|z_{k}\right|$

- The maximum height of the profile $R_{z}$, given by the difference between the maximum peak height $z_{\max }$ and the maximum valley depth $-z_{\text {min }}$.

The table II shows the values of these parameters for each test. The figure 13 and the table highlight the effectiveness of the correction realized in these tests. The correction of the stiffness error reduces $\mathrm{Ra}$ up to $77 \%$ and $\mathrm{Rz}$ up to $27 \%$. The correction of the kinematic error reduces Ra up to $32 \%$ and

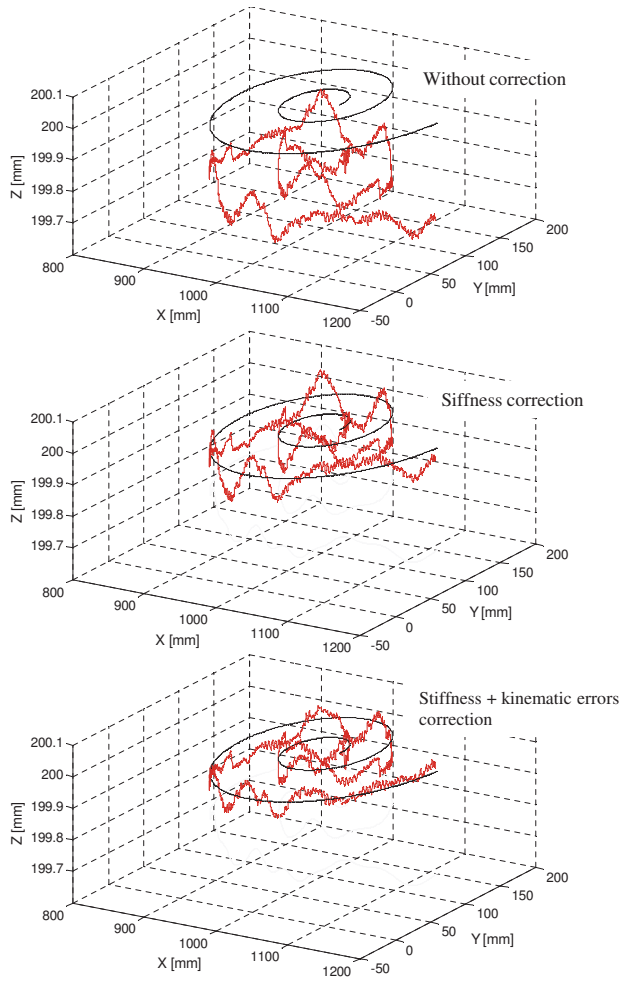

Fig. 13. measured trajectories without and with correction

Rz $28 \%$.

\section{CONCLUSION}

In this paper, two types of joints errors in industrial robots, joints compliances and kinematic errors, were presented. A local identification method, called axis by axis, is used in this work to identify the joints stiffness of a six axes industrial robot. An identification protocol to measure the kinematic error of joint 2 is presented in this work as well. Then an offline correction method is developed to improve the accuracy of industrial robots. This method is validated experimentally by measuring the trajectory of the end effector with and without correction to highlight its effectiveness.

\section{ACKNOWLEDGMENT}

The authors would like to thank STAUBLI Robotics for providing the necessary information and $\mathrm{Mr}$ X.Helle for his help with the experimental validations.

\section{REFERENCES}

[1] M. Damak, J. Grosbois, and P. De Smet, "Vision robot based absolute accuracy measurement, calibration and uncertainty." The 35th International Symposium on Robotics. Paris-NordVillepinte., 2004.

[2] H. Chen, T. Fuhlbrigge, S. Choi, J. Wang, and X. Li, "Practical industrial robot zero offset calibration," in 4th IEEE Conference on Automation Science and Engineering, CASE 2008, 2008, pp. 516-521.

[3] A. Elatta, G. Li Pei, Z. Fan Liang, D. Yu, and L. Fei, "An overview of robot calibration," in Information Technology Journal, CASE 2008, vol. 3, 2004, pp. 74-68.

[4] C. Gong, J. Yuan, and J. Ni, "Nongeometric error identification and compensation for robotic system by inverse calibration," International Journal of Machine Tools and Manufacture, vol. 40, no. 14, pp. 2119 2137, 2000. 
[5] E. Abele, M. Weigold, and S. Rothenbcher, "Modeling and identification of an industrial robot for machining applications," CIRP Annals Manufacturing Technology, vol. 56, no. 1, pp. 387-390, 2007.

[6] P. Lambrechts, M. Boerlage, and M. Steinbuch, "Trajectory planning and feedforward design for electromechanical motion systems," Control Engineering Practice, vol. 13, no. 2, pp. 145-157, 2005.

[7] W. B. J. Hakvoort, R. G. K. M. Aarts, J. van Dijk, and J. B. Jonker, "Lifted system iterative learning control applied to an industrial robot," Control Engineering Practice, vol. 16, no. 4, pp. 377-391, 2008.

[8] E. Abele, S. Bauer, S. Rothenbucher, M. Stelzer, and O. von Stryk, "Prediction of the tool displacement by coupled models of the compliant industrial robot and the milling process," in Proceedings of the International Conference on Process Machine Interactions, September 2008, 2008, pp. 223-230.

[9] E. Abele, J. Bauer, M. Pischan, O. v. Stryk, M. Friedmann, and T. Hemker, "Prediction of the tool displacement for robot milling applications using co-simulation of an industrial robot and a removal process," in CIRP 2nd International Conference Process Machine Interactions. CIRP, jun 2010.

[10] J. Wang, H. Zhang, and T. Fuhlbrigge, "Improving machining accuracy with robot deformation compensation," in 2009 IEEE/RSJ International Conference on Intelligent Robots and Systems, IROS 2009, 2009, pp. 3826-3831.

[11] A. Olabi, R. Bearee, E. Nyiri, and O. Gibaru, "Enhanced trajectory planning for machining with industrial six-axis robots," in Proceedings of the IEEE International Conference on Industrial Technology, 2010, pp. 500-506.

[12] A. Olabi, R. Bare, O. Gibaru, and M. Damak, "Feedrate planning for machining with industrial six-axis robots," Control Engineering Practice, vol. 18, no. 5, pp. 471-482, 2010.

[13] G. Alici and B. Shirinzadeh, "Enhanced stiffness modeling, identification and characterization for robot manipulators," IEEE Transactions on Robotics, vol. 21, no. 4, pp. 554-564, 2005.

[14] D. Tuttle, "Understanding and modeling the behavior of a harmonic drive gear transmission," Technical report, Massachusetts Institute of Technology, 1992.

[15] T. Wongratanaphisan and M. Chew, "Gravity compensation of spatial two-dof serial manipulators," Journal of Robotic Systems, vol. 19, no. 7, pp. 329-347, 2002

[16] F. H. Ghorbel, P. S. Gandhi, and F. Alpeter, "On the kinematic error in harmonic drive gears," Journal of Mechanical Design, Transactions Of the ASME, vol. 123, no. 1, pp. 90-97, 2001. 